\title{
A Search for Higher-Dimensional Integrable Modified KdV Equations - The Painlevé Approach
}

Kouichi TODA

Department of Physics, Keio University, Hiyoshi 4-1-1, Yokohama, 223-8521, JAPAN

Yukawa Institute for Theoretical Physics, Kyoto University, Kitashirakawa-Oiwake-Cho, Sakyo-ku, Kyoto 606-8502, JAPAN

E-mail: toda@phys-h.keio.ac.jp

Received April 30, 2001; Revised 21 December, 2001; Accepted 22 December, 2001

\begin{abstract}
It is shown here that the possibility of the existence of new $(2+1)$ dimensional integrable equations of the modified KdV equation using the Painlevé test.
\end{abstract}

\section{Introduction}

A central and active topic in the theory of integrable systems is to study as many higher dimensional integrable systems as possible. In this paper we will give $(2+1)$ dimensional integrable equations of the modified $\mathrm{KdV}(\mathrm{mKdV})$ equation via the Painlevé test. Let us first recall here that the $\mathrm{mKdV}$ equation in $(1+1)$ dimensions reads

$$
v_{t}+v_{x x x}+\frac{3}{2} v^{2} v_{x}=0
$$

where $v=v(x, t)$ and a subscript denotes partial differentiation, e.g., $v_{x}=\frac{\partial v}{\partial x}, v_{x x}=\frac{\partial^{2} v}{\partial x^{2}}$ etc. Higher dimensional integrable equations are not usually unique, in the sense that there exist several equations that reduce to a given one under dimensional reduction. It is known, for instance, that

$$
\begin{aligned}
& v_{t}+v_{x x z}+v^{2} v_{z}+v_{x}\left(\partial_{x}^{-1} v v_{z}\right)=0 \\
& \quad \text { and } \\
& v_{t}+v_{x x x}+\frac{3}{4} v_{x} \partial_{z}^{-1}\left\{v\left(\partial_{z}^{-1} v_{x}\right)_{x}\right\}+\frac{3}{4}\left(\partial_{z}^{-1} v_{x}\right)\left(v \partial_{z}^{-1} v_{x}\right)_{x} \\
& \quad-\frac{3}{4} v_{x}\left(\partial_{z}^{-1} v_{x}\right)^{2}=0
\end{aligned}
$$


are the higher-dimensional $\mathrm{mKdV}$ equations[1, 2, 3], where $v=v(x, z, t)$ and $\partial_{x}^{-1} v \equiv$ $\int v d x$. It is easy to check equation (2) and (3) are reduced to equation (1), setting $z=x$. Equation (2) has a generalized form given by

$$
v_{t}+v_{x x z}+A v^{2} v_{z}+B v_{x}\left(\partial_{x}^{-1} v v_{z}\right)+C v v_{x}\left(\partial_{x}^{-1} v_{z}\right)=0
$$

where $A, B$ and $C$ are numerical parameters. Only if

$$
A+B / 2+C \neq 0
$$

one can check setting $z=x$ reduces equation (4) to the mKdV equation, which is different from the coefficients of equation (1). However nobody knows whether equation(4) is integrable or not.

In [4], Ablowitz, Ramani and Segur presented the following conjecture: every nonlinear ordinary differential equation (NODE) obtained by an exact reduction of a completely integrable nonlinear partial differential equation (NPDE) possesses the Painlevé property, namely its general solution can have no movable singular points other than poles. They propose to exploit it as a test (the Painlevé test) whether a given NPDE is completely integrable. In [5] Weiss, Tabor and Carnevale proposed a more direct approach without recourse to the reduction to an NODE. Their test is to construct solutions to the NPDE having poles and sufficient number of arbitrary functions. It is an interesting problem to apply this integrability test to higher- dimensional equations. Our goal in this paper is to show equation (4) with (5) satisfies the Painlevé property possibly under some conditions on the numerical parameters $A, B$ and $C$.

This paper organized as follows. In Section 2 we investigate the conditions on the numerical parameters using so-called the WTC method. As a result, we will obtain 3 conditions or Case (i)-Case (iii). In Section 3 we briefly introduce the Soliton solution for Case (i). Section 4 is devoted to summary.

\section{A Search for integrability- The Painlevé Approach}

In this section we perform the Painlevé test as formulated by Weiss, Tabor and Carneval (so-called the WTC method) [5] for finding the conditions for three numerical parameters of equation (4). For that we need to rewrite equation (4) for taking away the term of $\partial_{x}^{-1}$ of it. A suitable system to be analyzed is

$$
\begin{aligned}
& u_{x}-B v v_{z}=0, \\
& w_{x}-C v_{z}=0, \\
& v_{t}+v_{x x z}+A v^{2} v_{z}+B u v_{x}+C v v_{x} w=0,
\end{aligned}
$$

with the condition

$$
A+B / 2+C \neq 0
$$

where $u=u(x, z, t), v=v(x, z, t)$ and $w=w(x, z, t)$. The Painlevé test essentially amounts to find solutions to equations (6), (7) and (8) having the forms

$$
u=\sum_{j=0} u_{j} \phi^{j+\alpha}, \quad v=\sum_{j=0} v_{j} \phi^{j+\beta}, \quad w=\sum_{j=0} w_{j} \phi^{j+\gamma}
$$


with the movable singularity manifold determined by

$$
\phi=\phi(x, z, t)=0, \quad \phi_{x} \neq 0,
$$

where $u_{j}=u_{j}(x, z, t), v_{j}=v_{j}(x, z, t), w_{j}=w_{j}(x, z, t), u_{0} \neq 0, v_{0} \neq 0, w_{0} \neq 0$ and $\alpha-\gamma$ are negative integers (so-called leading order). Here $\phi$ and $u_{j}(x, z, t)-w_{j}(x, z, t)$ are analytic functions of $(x, z, t)$ in a neighborhood of the manifold (11). Note that the solutions (10) contain a sufficient number of arbitrary functions. By the leading order analysis, namely requiring

$$
u \sim u_{0} \phi^{\alpha}, \quad v \sim v_{0} \phi^{\beta}, \quad w \sim w_{0} \phi^{\gamma},
$$

we obtain

$$
\alpha=-2, \quad \beta=\gamma=-1,
$$

as leading orders with

$$
u_{0}=\frac{B}{2} \frac{\phi_{z}}{\phi_{x}} v_{0}^{2}, \quad w_{0}=C \frac{\phi_{z}}{\phi_{x}} v_{0} \quad \text { and } \quad v_{0}^{2}=-\frac{6 \phi_{x}^{2}}{A+B / 2+C} .
$$

Thus the substitution of the solutions (10) with (13) into equations (6)-(8) leads to three recursion formula. And then collecting terms of the formula involving $u_{j}-w_{j}$, it is found that

$$
\begin{aligned}
& (j-2) \phi_{x} u_{j}-B(j-2) v_{j}=f_{1}, \\
& (j-1) \phi_{x} w_{j}-C(j-1) \phi_{z} v_{j}=f_{2}, \\
& v_{0} \phi_{x} u_{j}-G(j) v_{j}+v_{0}^{2} \phi_{x} w_{j}=f_{3},
\end{aligned}
$$

where

$$
G(j)=\frac{12 A+6 C+j(A+B / 2+C)(j-1)(j-5)}{A+B / 2+C}
$$

and $f_{i}(i=1,2,3)$ is each a function of $u_{j-1}, \cdots, u_{0}, v_{j-1}, \cdots, v_{0}, w_{j-1}, \cdots, w_{0}$ and $\phi$. In matrix form, equations (15)-(17) are written as

$$
\left[\begin{array}{ccc}
(j-2) \phi_{x} & -B(j-2) & 0 \\
0 & -C(j-1) \phi_{z} & (j-1) \phi_{x} \\
v_{0} \phi_{x} & -G(j) & v_{0}^{2} \phi_{x}
\end{array}\right]\left(\begin{array}{c}
u_{j} \\
v_{j} \\
w_{j}
\end{array}\right)=\left(\begin{array}{c}
f_{1} \\
f_{2} \\
f_{3}
\end{array}\right) .
$$

Resonances, which are values of $j$ for which the recursion formula is not defined, occurs when

$$
\operatorname{det}\left|\begin{array}{ccc}
(j-2) \phi_{x} & -B(j-2) & 0 \\
0 & -C(j-1) \phi_{z} & (j-1) \phi_{x} \\
v_{0} \phi_{x} & -G(j) & v_{0}^{2} \phi_{x}
\end{array}\right|=0 .
$$

Trivial algebra yields the resonances

$$
j= \pm 1,2,3,4 \text {. }
$$

The resonance $j=-1$ in (21) corresponds to the arbitrary singularity manifold $\phi=$ 0 . To complete the Painlevé test one must verify the compatibility conditions at the 
resonances. Explicitly this means that equations (15)-(17) must vanish identically with no constraints on the arbitrary functions $\phi$ and one of $\left\{u_{j}, v_{j}, w_{j}\right\}$ associated with $j=1,2,3,4$ respectively . To simplify the calculations, we used the reduced manifold ansatz of Kruskal (see [6] for details);

$$
\phi=x+\rho(z, t), \quad u_{j}=u_{j}(z, t), \quad v_{j}=v_{j}(z, t) \text { and } w_{j}=w_{j}(z, t) .
$$

At this point, we have narrowed down our investigation of possible the Painlevé property to the following:

Case (i) $A=B \neq 0$ : The resonance conditions at $j=1,2,3,4$ require that one of $\left\{u_{1}, v_{1}, w_{1}\right\}$, one of $\left\{u_{2}, v_{2}, w_{2}\right\}$, one of $\left\{u_{3}, v_{3}, w_{3}\right\}$ and one of $\left\{u_{4}, v_{4}, w_{4}\right\}$ should be arbitrary respectively. This case corresponds to equation (2), which is different from the coefficients.

Case (ii) $A=B+C$ and $3 B+4 C \neq 0$ : The resonance conditions at $j=1,2,4$ require that one of $\left\{u_{1}, v_{1}, w_{1}\right\}$, one of $\left\{u_{2}, v_{2}, w_{2}\right\}$ and one of $\left\{u_{4}, v_{4}, w_{4}\right\}$ should be arbitrary respectively. And $v_{3}$ can be chosen an arbitrary function corresponding to $j=3$.

Case (iii) $A=B+C / 2$ and $B+C \neq 0$ : The resonance conditions at $j=1,3,4$ require that one of $\left\{u_{1}, v_{1}, w_{1}\right\}$, one of $\left\{u_{3}, v_{3}, w_{3}\right\}$ and one of $\left\{u_{4}, v_{4}, w_{4}\right\}$ should be arbitrary respectively. And $v_{2}$ can be chosen an arbitrary function corresponding to $j=2$.

We used MATHEMATICA to handle the calculation for the existence of arbitrary functions. Therefore equation (4) has the Painlevé property only for above parametric restriction Case (i)-Case (iii).

\section{Soliton solution of Case (i)}

For Case (i) or $A=B$, equation (4) is written as

$$
v_{t}+v_{x x z}+A v^{2} v_{z}+A v_{x}\left(\partial_{x}^{-1} v v_{z}\right)=0
$$

with $A$ being a non-zero numerical parameter.

This equation has Soliton solution [2]. Let us in this section mention briefly them.

For that we describe this equation in terms of the coupled system,

$$
\begin{aligned}
& \rho_{x}+A v^{2}=0, \\
& v_{t}+v_{x x z}-\rho_{x} v_{z}-\frac{1}{2} v_{x} \rho_{z} .
\end{aligned}
$$

By the transformation of the dependent variables

$$
\begin{gathered}
v=\left[\log \left(\frac{F}{G}\right)\right]_{x} \\
\text { and } \\
\rho=[\log (F G)]_{x},
\end{gathered}
$$


then equations (24) and (25) are reduced to the bilinear forms [2],

$$
\begin{aligned}
& \mathcal{D}_{x}^{2} F \cdot G=0 \\
& \quad \text { and } \\
& \left(\mathcal{D}_{t}-\mathcal{D}_{x}^{2} D_{z}\right) F \cdot G=0,
\end{aligned}
$$

where Hirota's derivative $\mathcal{D}$ operating on $F \cdot G$ is defined by

$$
\left.\mathcal{D}_{x}^{n} F(x) \cdot G(x) \equiv\left(\partial_{x_{1}}-\partial_{x_{2}}\right)^{n} F\left(x_{1}\right) G\left(x_{2}\right)\right|_{x_{1}=x_{2}=x} .
$$

$N$ soliton solution to equations (28) and (29) are speculated from the conventional Hirota's direct method,

$$
\begin{aligned}
& F=1+\sum_{n=1}^{N} \sum_{N} C_{n} \eta_{i_{1} \cdots i_{n}} \exp \left(\lambda_{i_{1}}+\cdots+\lambda_{i_{n}}\right), \\
& G=1+\sum_{n=1}^{N} \sum_{N}(-1)^{n} \eta_{i_{1} \cdots i_{n}} \exp \left(\lambda_{i_{1}}+\cdots+\lambda_{i_{n}}\right), \\
& \lambda_{j}=p_{j} x+q_{j} z+r_{j} t+s_{j} \\
& q_{j}=p_{j}^{2} q_{j} \\
& \eta_{j, k}=\frac{\left(p_{j}-p_{k}\right)^{2}}{\left(p_{j}+p_{k}\right)^{2}} \\
& \eta_{i_{1} \cdots i_{n}}=\eta_{i_{1}, i_{2}} \cdots \eta_{i_{1}, i_{n}} \cdots \eta_{i_{n-1}, i_{n}} .
\end{aligned}
$$

where $p_{j}, q_{j}, r_{j}$ and $s_{j}$ are arbitrary constants. In $[1,7,8]$, it was shown that equation (23) has also the Lax pair.

\section{Summary}

The present analysis shows that equation (4) passes the Painlevé test in the sense of WTC method with Kruskal's ansatz only under Case (i)-Case (iii). Case (i) corresponds to equation (2). Except for Case (i), the Lax pair, Hirota's bilinear form and Soliton solution for equations of Case (ii) and Case (iii) have not been constructed yet. Further study on this topic continues.

\section{Acknowledgement}

The author would like to express his sincere thanks to the editors, especially Dr. W.-X. Ma for his helpful comments. He is grateful for hospitality the Yukawa Institute, where the paper was partly prepared during his visit. Many valuable discussions with Professor J. Hietarinta, Professor X.-B. Hu, Dr. T. Ikeda, Professor K. Konno, Professor M. Omote, Professor R. Sasaki and Dr. S.-J. Yu are also acknowledged. This work was financially supported by the Sasagawa Scientific Research Grant from The Japan Science Society. 


\section{References}

[1] Bogoyavlensky O I, Maths. USSR. Izv. 34, 245 (1990).

[2] Yu S-J, Toda K, Sasa N and Fukuyama T, J. Phys. A 31, 3337 (1998).

[3] Toda $\mathrm{K}$ and $\mathrm{Yu}$ S-J, in preparation.

[4] Ablowitz M J, Ramani A and Segur H, J. Maths. Phys. 21, 715 (1980).

[5] Weiss J, Tabor M J and Carnevale G, J. Maths. Phys. 24, 522 (1983).

[6] Kruskal M D, Joshi N and Halburd R, solv-int/9710023

[7] Yu S-J, Toda K and Fukuyama T, J. Phys. A 31, 10181 (1998).

[8] Toda K, Yu S-J and Fukuyama T, Reps. Maths. Phys. 44, 256 (1999). 